\title{
RESEARCH
}

Open Access

\section{Genetically determined blood pressure, antihypertensive medications, and risk of Alzheimer's disease: a Mendelian randomization study}

Ya-Nan Ou', Yu-Xiang Yang ${ }^{2}$, Xue-Ning Shen ${ }^{2}$, Ya-Hui Ma', Shi-Dong Chen², Qiang Dong ${ }^{2 *}$, Lan Tan ${ }^{1 *}$ and Jin-Tai $Y u^{2^{*}}$

\begin{abstract}
Background: Observational studies suggest that the use of antihypertensive medications (AHMs) is associated with a reduced risk of Alzheimer's disease (AD); however, these findings may be biased by confounding and reverse causality. We aimed to explore the effects of blood pressure (BP) and lowering systolic BP (SBP) via the protein targets of different AHMs on AD through a two-sample Mendelian randomization (MR) approach.

Methods: Genetic proxies from genome-wide association studies of BP traits and BP-lowering variants in genes encoding AHM targets were extracted. Estimates were calculated by inverse-variance weighted method as the main model. MR Egger regression and leave-one-out analysis were performed to identify potential violations.

Results: There was limited evidence that genetically predicted SBP/diastolic BP level affected AD risk based on 400/ 398 single nucleotide polymorphisms (SNPs), respectively (all $P>0.05$ ). Suitable genetic variants for $\beta$-blockers (1 SNP), angiotensin receptor blockers (1 SNP), calcium channel blockers (CCBs, 45 SNPs), and thiazide diuretics (5 SNPs) were identified. Genetic proxies for CCB [odds ratio $(\mathrm{OR})=0.959,95 \%$ confidence interval $(C \mathrm{Cl})=0.941-0.977$, $\left.P=3.92 \times 10^{-6}\right]$ and overall use of AHMs $\left(\mathrm{OR}=0.961,95 \% \mathrm{Cl}=0.944-0.978, P=5.74 \times 10^{-6}, \mathrm{SNPs}=52\right)$ were associated with a lower risk of AD. No notable heterogeneity and directional pleiotropy were identified (all $P>0.05)$. Additional analyses partly support these results. No single SNP was driving the observed effects.
\end{abstract}

Conclusions: This MR analysis found evidence that genetically determined lowering BP was associated with a lower risk of $A D$ and $C C B$ was identified as a promising strategy for $A D$ prevention.

Keywords: Blood pressure, Antihypertensive medications, Alzheimer's disease, Single nucleotide polymorphism, Mendelian randomization

\footnotetext{
*Correspondence: dong_qiang@fudan.edu.cn; dr.tanlan@163.com; jintai_yu@fudan.edu.cn

${ }^{2}$ Department of Neurology and Institute of Neurology, WHO Collaborating Center for Research and Training in Neurosciences, Huashan Hospital,

Shanghai Medical College, Fudan University, Shanghai, China

'Department of Neurology, Qingdao Municipal Hospital, Qingdao University,

Qingdao 266071, China
}

C C The Author(s). 2021 Open Access This article is licensed under a Creative Commons Attribution 4.0 International License, which permits use, sharing, adaptation, distribution and reproduction in any medium or format, as long as you give appropriate credit to the original author(s) and the source, provide a link to the Creative Commons licence, and indicate if changes were made. The images or other third party material in this article are included in the article's Creative Commons licence, unless indicated otherwise in a credit line to the material. If material is not included in the article's Creative Commons licence and your intended use is not permitted by statutory regulation or exceeds the permitted use, you will need to obtain permission directly from the copyright holder. To view a copy of this licence, visit http://creativecommons.org/licenses/by/4.0/ The Creative Commons Public Domain Dedication waiver (http://creativecommons.org/publicdomain/zero/1.0/) applies to the data made available in this article, unless otherwise stated in a credit line to the data. 


\section{Background}

Alzheimer's disease (AD) prevalence is rising, further increasing the social and economic burden [1]. In the absence of any therapeutic intervention, prevention strategies that target modifiable risk factors are promising approaches. Hypertension has emerged as a potential risk factor for AD $[2,3]$. Antihypertensive medications (AHMs) have also been highlighted as priority repurposing candidates for $\mathrm{AD}$ prevention [4, 5]. However, inference from observational studies is limited by residual confounding, reverse causation, and detection bias [6]. Difficulties in implementing large-scale randomized clinical trials (RCTs) also restrict the exploration of this association.

A novel method for estimating causal effects of risk factors in observational studies using genetic variants is Mendelian randomization (MR) [7]. Due to the random assortment of genes at conception, MR overcomes the core shortcomings of observational studies and assesses lifelong exposures to risk factors, and thus, it can clarify potential causal associations [6]. Recently, two studies estimated causal effects of BP level on AD risk using MR analysis. One employed 24 single nucleotide polymorphisms (SNPs) indicating that genetically predicted higher systolic blood pressure (SBP) was causally associated with a lower risk of $\mathrm{AD}$ [8]. However, another study exploited more SNPs $(N=93)$, finding no significant association between SBP level and AD [9]. Andrews et al. also found a null association between polygenic risk score (PRS, prioritizing putative causal risk factor score) for increased SBP and $\mathrm{AD}$ risk [10]. The differences among the research results may lie in the differences in the number of SNPs included, the statistical power, and analytical bias; thus, higherquality studies with larger sample size are urgently needed to corroborate this question. Furthermore, the expression and function of drug targets can be influenced by variants within or near the genes that encode them. Therefore, the effects of drug action can be anticipated by the genetic effects in the genes of their protein targets, as has previously been applied to lipid-lowering drugs [11]. However, only one previous study employed MR approach to investigate the effect of AHMs on AD risk, suggesting that lowering SBP via the protein targets of AHMs is unlikely to affect the risk of developing AD [12].

Herein, considering that previous MR analyses have yielded opposite results and larger databases of BP traits and $\mathrm{AD}$ are now available, we aimed to perform a twosample MR analysis to assess the causal effects of genetically determined $\mathrm{BP}$ and genetic proxies for antihypertensive drug classes on the risk of AD comprehensively.

\section{Methods}

\section{Instrument identification}

Significant SNPs $\left(P<5 \times 10^{-8}\right)$ for BP were identified from a genome-wide association study (GWAS) meta- analysis that included 757,601 individuals of European ancestry drawn from UK Biobank (UKB, $N=458,577$ ) and the International Consortium of Blood Pressure (ICBP, $N=299,024)$ database [13]. In UKB, two BP measurements were taken after a 2-min rest using an Omron HEM-7015IT digital BP monitor, or a manual sphygmometer. The mean SBP and diastolic BP (DBP) values were calculated from two automated or two manual BP measurements. For individuals with one manual and one automated BP measurement, the mean of these two values were used. For individuals with only one available BP measurement, this single value was used. The mean age of participants ranged from 56.8 to 62.1 years old (see Additional file 1).

We selected genetic variants as proxies for the SBP lowering effects of common antihypertensive drug classes: angiotensin converting enzyme inhibitors (ACEI), angiotensin receptor blockers (ARB), $\beta$ blockers (BB), calcium channel blockers (CCB), and thiazide diuretic agents on the basis of new consensus guidelines (Fig. 1) [14]. We identified the genes encoding pharmacologic targets related to BP lowering for common antihypertensive drug classes in DrugBank (https://www.drugbank.ca/) [15] and screened the genomic SNPs corresponding to these genes in GeneCards (https://www.genecards.org/) [16]. From all the identified variants in each gene, only variants that are significantly associated with SBP $\left(P<5 \times 10^{-8}\right)$ and clumped to a linkage disequilibrium (LD) threshold of $R^{2}<0.4$ using the $1000 \mathrm{G}$ European reference panel were considered as candidate proxies for each medication class. This relatively lenient LD correlation threshold allows for an increase in proportion of variance explained and thus in statistical power [17-19]. For additional analysis, we also employed more stringent LD thresholds $\left(R^{2}<0.1\right.$ and $R^{2}<0.001$, respectively).

\section{AD database}

AD data were from a large famous GWAS dataset from International Genomics of Alzheimer's Project (IGAP) GWAS Stage 1 result ( $N=21,982$ cases, 41,944 controls) [20]. It is composed of datasets from the Alzheimer Disease Genetics Consortium (ADGC), Cohorts for Heart and Aging Research in Genomic Epidemiology Consortium (CHARGE), The European Alzheimer's Disease Initiative (EADI), and Genetic and Environmental Risk in AD/Defining Genetic, Polygenic and Environmental Risk for Alzheimer's Disease Consortium (GERA D/PERADES). AD cases were all autopsy-confirmed or clinically confirmed using published criteria. The mean age of onset of AD cases ranged from 71.1 to 82.6 years, and the mean age of onset of healthy controls ranged from 51.0 to 78.9 years (see Additional file 1). 


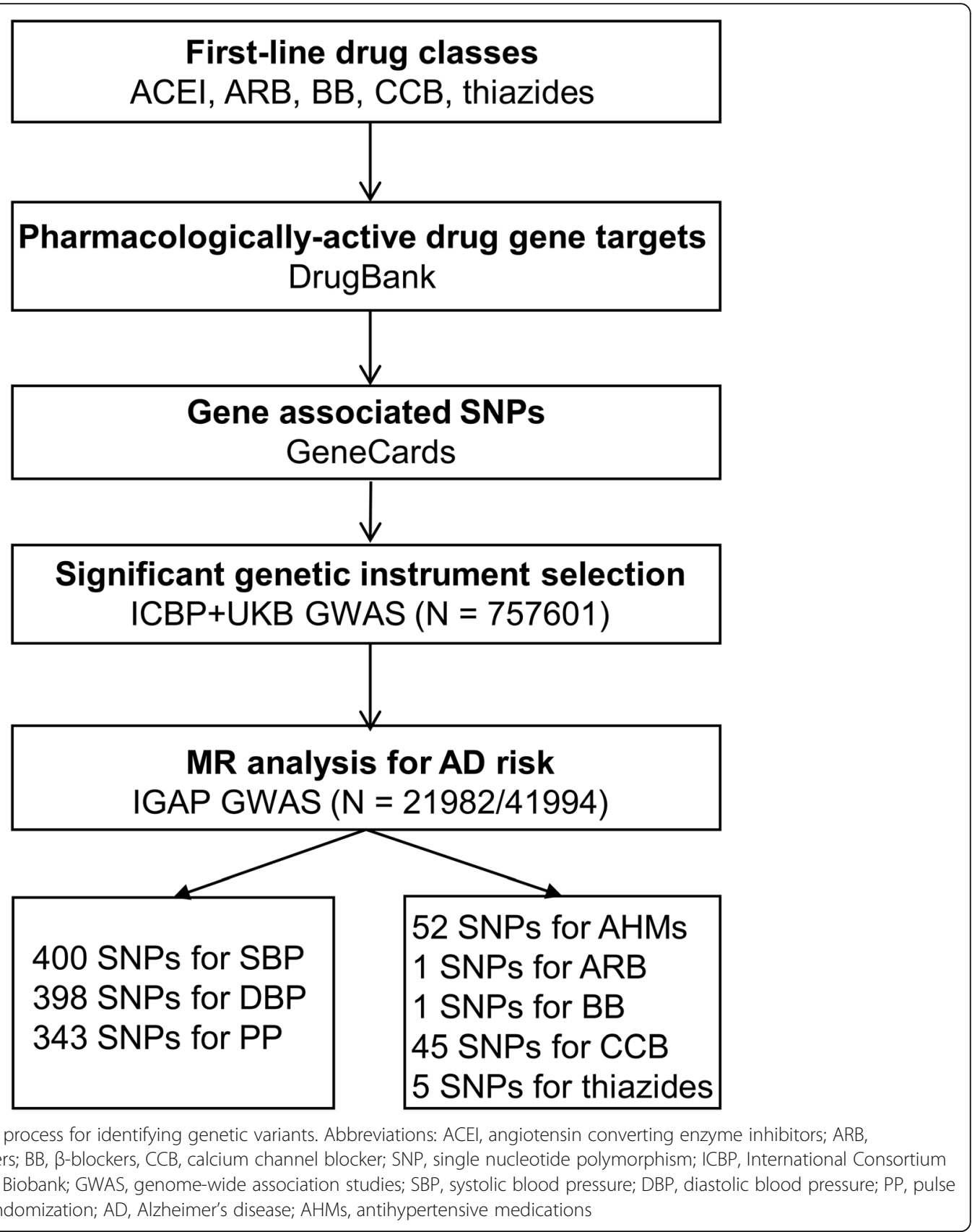

\section{Statistical analyses}

This MR approach was based on 3 assumptions: (1) the genetic variants associate with the exposure, (2) the instrumental variables (IVs) have no association with confounding factors, and (3) the risk of AD is influenced only by the exposure, not by other pathways [7, 21]. We estimated the overall effect of SBP/DBP/pulse pressure (PP) on $\mathrm{AD}$ by combining the effects of all the genomewide significant SNPs $\left(P<5 \times 10^{-8}\right)$ from the UKB+ICBP GWAS which were then clumped based on the European 1000 Genomes panel to $R^{2}<0.001$. SNPs absent in the outcome data were replaced by proxy SNPs in high LD from the 1000 Genomes Project European data where possible. Proxies were required to have a minimum $R^{2}$ value of 0.8 and palindromic SNP strands were aligned using minor allele frequency up to 0.3 [22]. We excluded the SNPs that had $F$ statistics lower than 10 according to standard practice [23]. Then, we estimated the effects of AHMs on AD by selecting SNPs associated with AHMs at genome-wide significance $\left(P<5 \times 10^{-8}\right)$ that were at moderate to low $\operatorname{LD}\left(R^{2}<0.4\right)$ and more stringent LD thresholds $\left(R^{2}<0.1\right.$ and $\left.R^{2}<0.001\right)$. The 
genes and the specific genomic regions screened for the identification of genetic proxies for each AHM class were detailed (see Additional file 2).

Causal effects were estimated with the random-effects maximum likelihood estimation method. We applied five complementary methods [inverse variance weighted (IVW), MR-Egger, weighted median, simple mode, and weighted mode], which provided different assumptions about horizontal pleiotropy [24]. The IVW method was performed as our primary method, which essentially assumed the intercept was zero and associated a weighted regression of SNP-exposure effects with SNP-outcome effects. The weighted median approaches give more weight to more precise instrumental variables and the estimate is consistent even when up to $50 \%$ of the information comes from invalid or weak instruments [7]. The association is considered significant after the correction for multiple testing for three BP indexes $[P<0.016$ $(0.05 / 3)]$ and five AHM classes $[P<0.01(0.05 / 5)]$. A $P$ value above $0.016 / 0.01$ but below 0.05 was considered suggestive of evidence for a potential association. Results were presented as odds ratios (ORs) and 95\% confidence intervals (CIs) of AD per genetically predicted unit logtransformed increase in each trait. We estimated the intercept of MR-Egger regression, which represented the average horizontal pleiotropy [25]. We conducted a leave-one-SNP-out analysis in which we systematically removed one SNP at a time to assess the influence of potentially pleiotropic SNPs on the causal estimates. The strength of the genetic instrument was judged with $F$ statistics [23]. $F$ statistics are greater than 10, indicating that the instrument strength was sufficient for MR analysis [26]. Statistical significance of the above analyses was set at a 2 -sided $P$ value $<0.05$. Statistical analyses were conducted in $\mathrm{R}$ (version 3.5.3) and MR analyses were conducted using "TwoSampleMR".

\section{Results}

\section{Genetically determined $B P$ and risk of $A D$}

First, we examined the relationship between genetically determined BP and the risk of AD. A total of 400/398/ 343 independent genetic variants were found to be associated with SBP/DBP/PP, respectively (see Additional files $3,4,5$ ). There was no evidence of an association between either genetically predicted SBP or PP with $\mathrm{AD}$, with $P$ values $>0.05$ in all of the analyses (Fig. 2). However, the results were suggestive of an association between DBP and AD using IVW method with an OR of 0.990 (95\% CI $=0.979-1.000, P=0.055)$. The sensitivity analyses, MR Egger (OR $=0.973,95 \%$ $\mathrm{CI}=0.947-1.000, \quad P=0.047), \quad$ and weighted median $(\mathrm{OR}=0.985,95 \% \mathrm{CI}=0.970-1.001, P=0.062)$ methods confirmed these results. There was evidence of heterogeneity in the causal effect estimates from all of the MR analyses (all $P$ values $<0.05$, Table 1 ). Nonetheless, horizontal pleiotropic effects were absent in MR Egger

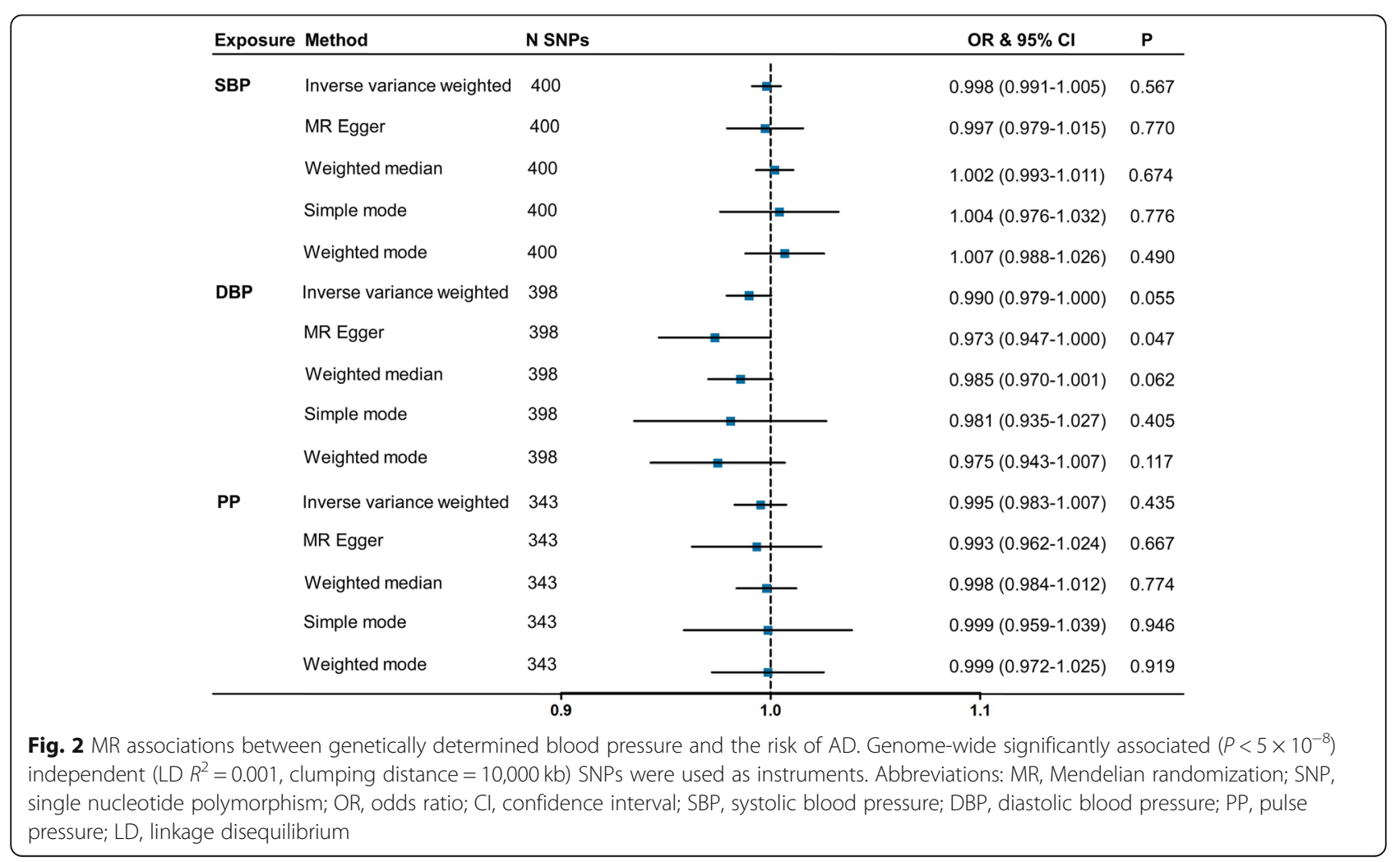


Table 1 Heterogeneity and pleiotropy tests of instrument effects

\begin{tabular}{|c|c|c|c|c|c|c|c|c|}
\hline \multirow[t]{2}{*}{ Exposure } & \multirow[t]{2}{*}{ N SNPs } & \multicolumn{4}{|c|}{ Heterogeneity analysis } & \multicolumn{3}{|c|}{ Pleiotropy analysis } \\
\hline & & Method & $\mathbf{Q}$ & Degree of freedom & $\mathbf{P}$ & Egger intercept & SE & $\mathbf{P}$ \\
\hline \multirow[t]{2}{*}{$\mathrm{CCB}$} & 45 & MR Egger & 26.07 & 43 & 0.981 & $-2.19 \times 10^{-3}$ & 0.009 & 0.806 \\
\hline & & IVW & 26.13 & 44 & 0.985 & & & \\
\hline \multirow[t]{2}{*}{ Thiazides } & 5 & MR Egger & 2.55 & 3 & 0.466 & $-4.42 \times 10^{-2}$ & 0.041 & 0.350 \\
\hline & & IVW & 3.77 & 4 & 0.438 & & & \\
\hline \multirow[t]{2}{*}{ AHMs } & 52 & MR Egger & 31.73 & 50 & 0.980 & $-4.54 \times 10^{-3}$ & 0.009 & 0.586 \\
\hline & & IVW & 32.03 & 51 & 0.983 & & & \\
\hline \multirow[t]{2}{*}{ SBP } & 400 & MR Egger & 635.41 & 398 & $3.506 \times 10^{-13}$ & $2.27 \times 10^{-4}$ & 0.003 & 0.935 \\
\hline & & IVW & 635.42 & 399 & $4.441 \times 10^{-13}$ & & & \\
\hline \multirow[t]{2}{*}{ DBP } & 398 & MR Egger & 524.27 & 396 & $1.557 \times 10^{-5}$ & $3.25 \times 10^{-3}$ & 0.002 & 0.182 \\
\hline & & IVW & 526.63 & 397 & $1.330 \times 10^{-5}$ & & & \\
\hline \multirow[t]{2}{*}{ PP } & 343 & MR Egger & 699.38 & 341 & $6.704 \times 10^{-27}$ & $4.24 \times 10^{-4}$ & 0.003 & 0.897 \\
\hline & & IVW & 699.41 & 342 & $9.550 \times 10^{-27}$ & & & \\
\hline
\end{tabular}

Abbreviations: MR Mendelian randomization, $I V W$ inverse variance weighted, $C C B$ calcium channel blocker, $A H M s$ antihypertensive medications, $S B P$ systolic blood pressure, $D B P$ diastolic blood pressure, $P P$ pulse pressure, $S E$ standard error

regression (intercept $0.003, P=0.935$ for $\mathrm{SBP}$; intercept $0.002, P=0.182$ for DBP; intercept $0.003, P=0.897$ for PP; Table 1). We did not find a single genetic variant of BP that had an influence on the association in the leaveone-out analysis.

Genetic proxies for antihypertensive drugs and risk of $A D$ Next, we selected BP-lowering variants in genes encoding drug targets as proxies for the effects of AHM classes and examined their effects on AD. We identified a total of 52 variants for AHMs, including 1 for ARB, 1 for BB, 45 for $\mathrm{CCB}$, and 5 for thiazides. However, we failed to explore the casual effects of ACEI on AD risk because no proxy was identified. All SNPs had $F$ values $>10$, suggesting that they were unlikely to introduce marked weak instrument bias into the MR analyses (see Additional file 6).

The MR analysis showed an association of the overall use of AHMs with lower risk of AD (OR $=0.961,95 \%$ $\mathrm{CI}=0.944-0.978, P=5.74 \times 10^{-6}, \mathrm{SNPs}=52$; Fig. 3$)$. The associations were confirmed using sensitivity analyses including the methods of weighted median ( $\mathrm{OR}=0.961$, 95\% CI $=0.937-0.985, P=0.001)$, simple mode $(\mathrm{OR}=$ 0.949, 95\% CI $=0.903-0.996, P=0.032)$, and weighted mode $(\mathrm{OR}=0.958, \quad 95 \% \quad \mathrm{CI}=0.921-0.996, \quad P=0.031)$. The Cochran $Q$ statistic of IVW method $(Q=26.130$; $P=0.985)$ indicated no notable heterogeneity across instrument SNP effects (Table 1). Egger analysis did not show evidence of directional pleiotropy $(P>0.05)$. There was no distortion in the leave-one-out plot, suggesting that no single SNP was driving the observed effect in any analysis (see Additional file 7).

Reduction in SBP through variants in genes encoding targets of $\mathrm{CCB}$ was associated with a lower risk of $\mathrm{AD}$
(OR $=0.959, \quad 95 \% \quad \mathrm{CI}=0.941-0.977, \quad P=3.92 \times 10^{-6}$, SNPs $=45$; Fig. 3). The association was confirmed by sensitivity analyses using weighted median method $(\mathrm{OR}=0.960,95 \% \mathrm{CI}=0.935-0.985, P=0.001)$. No evidence of heterogeneity and pleiotropy in the causal effect estimates was found (all $P>0.05$, Table 1 ). Leaveone-out analysis did not change the overall direction (see Additional file 7). However, we did not find evidence of causal effects of $A R B, B B$, and thiazides on the risk of $\mathrm{AD}$.

\section{Results of additional analysis}

Additional analysis restricted to the set of SNPs with the LD threshold $\left(R^{2}<0.1\right)$ showed consistent association estimates with an OR of 0.968 (95\% CI $=0.942-0.994$, $P=0.003$, SNPs $=27$; see Additional file 8 ) for the overall AHMs. However, none of the sensitivity analyses were significant, including weighted median method with $P=$ 0.078. Then, corresponding estimates using $R^{2}<0.001$ were also presented, and estimates were partly consistent with a borderline significant $\mathrm{OR}_{\mathrm{WM}}$ of $0.951(P=0.063$, SNPs = 9; see Additional file 9). As for CCB, the results were consistent using LD $R^{2}<0.1$ with 21 SNPs included, resulting in an OR of 0.955 (95\% CI $=0.927-$ 0.983, $P=0.002$ ). Weighted median analysis failed to support the positive association with $P=0.099$. When the more stringent pairwise threshold $\left(R^{2}<0.001\right)$ was used to construct the aggregated IVs, the results were partly concordant with the main observations with $P_{\mathrm{WM}}=0.096$. These relationships were again not robust, thus highlighting the need for caution in interpreting these effects as causal. 


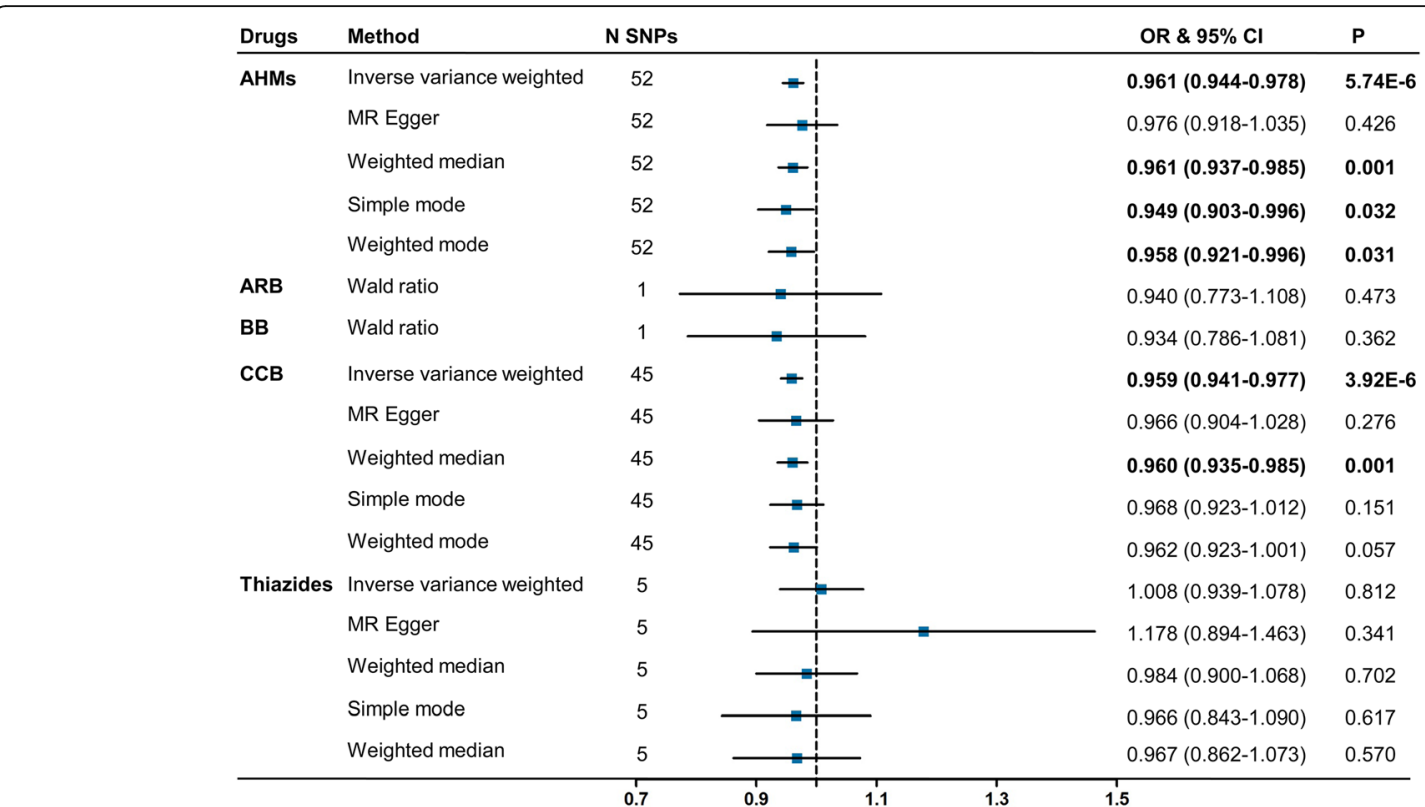

Fig. 3 MR associations between genetic proxies for antihypertensive drug classes and the risk of AD. Genome-wide significantly associated $(P<$ $5 \times 10^{-8}$ ) independent (LD $\left.R^{2}<0.4\right)$ SNPs were used as instruments. Bold fonts indicate significant associations. Abbreviations: SNP, single nucleotide polymorphism; OR, odds ratio; $\mathrm{Cl}$, confidence interval; $\mathrm{AHMs}$, antihypertensive medications; $\mathrm{ARB}$, angiotensin receptor blockers; $\mathrm{BB}$, $\beta$ blockers; CCB, calcium channel blocker; LD, linkage disequilibrium

\section{Discussion}

This two-sample MR study, in which we used genetic variants as proxies associated with BP in a very large cohort of well-characterized research participants, provided suggestive evidence for associations between genetically exposure to BP lowering through AHMs and a reduced risk of $\mathrm{AD}$ and further identified $\mathrm{CCB}$ as a promising strategy for $\mathrm{AD}$ prevention. However, these results should be interpreted with great caution.

Hypertension has been implicated as a risk factor for $\mathrm{AD}$ [3]. However, uncertainties remain over the nature of the association, which perhaps is complicated by misclassification of different forms of dementia, or the age of study participants [2, 27]. Several studies suggested that high $\mathrm{BP}$ in midlife was associated with a higher risk of $\mathrm{AD}$, whereas other studies indicated that high $\mathrm{BP}$ in late life might be protective against $\mathrm{AD}[2,27,28]$. Large-scale biobank datasets can provide an unparalleled opportunity to undertake hypothesis-free causal inference. Using a MR approach, the current study failed to identify a causal relationship between SBP level and AD risk. Previous observational studies have produced a consistent finding of no association between high blood pressure and AD in late-life [28]. There are two earlier studies using MR to evaluate the association of SBP with AD cases-control status. Østergaard and colleagues observed that higher SBP was associated with a reduced risk of $\mathrm{AD}$ [8]. However, Larsson and colleagues exploited more SNPs, finding no significant association between SBP level and AD [9]. Andrews and colleagues also found a null association between PRS for increased SBP and AD risk [10].

The association of DBP level with clinically diagnosed $\mathrm{AD}$ has not been extensively studied, though several studies have conducted phenome-wide scans. Using data from the UK Biobank, Richardson and colleagues found that a higher AD PRS was associated with lower DBP [29]. Similarly, a second study by Korologou-Linden and colleagues evaluated the association of an AD PRS composed of 18 SNPs, inclusive of $A P O E$, finding that a higher AD PRS was associated with lower DBP [30]. This present result was also suggestive of an association between high DBP level and a lower risk of AD. High BP in late life might be protective against $\mathrm{AD}$. Consistent with this, one study also showed a greater effect of decreased DBP on white matter hyperintensity volume (WMHV) burden, particularly among those who previously had a greater increase in SBP [31]. Hypotension in late life might aggravate cerebral small vessel disease and decrease brain volume in cognitively normal individuals, potentially via shifts in the auto-regulatory curve and resultant cerebral hypoperfusion [32, 33]. Alternatively, this finding might be mediated by increased arterial stiffness, which is associated with decreased DBP, although we did not find a direct association between PP, a proxy marker of arterial stiffness, and AD risk. One recent MR analysis observed association between high BP and vascular brain injury (VBI), which suggests that while 
reducing $\mathrm{BP}$ in late life may have limited utility in the prevention of $\mathrm{AD}$, it may reduce the risk of vascular dementia by reducing the risk of VBI, but not specifically affect the risk of $\mathrm{AD}[10]$.

There is also a wealth of evidence in the literature from observational studies indicating that antihypertensive therapy may protect against $\mathrm{AD}$ or delay its onset $[4,5]$. A recent high-quality meta-analysis found that among people with high BP, the use of AHMs might reduce the risk of $\mathrm{AD}$ [4]. A British cohort study of dementia-free individuals concluded that BP monitoring and interventions need to start around 40 years of age to preserve cognition in older age [31]. However, the wellknown SPRINT MIND study did not find any significant difference in the risk of dementia between intensive and standard BP control [34]. The study may have been underpowered for this end point due to early study termination and fewer than expected cases of dementia. Using MR, the current study extends previous evidence using genetic variants in a very large cohort of wellcharacterized research participants and then selected gene targets of AHMs, showing that genetically determined lowering BP through AHMs was associated with a lower risk of $\mathrm{AD}$, and $\mathrm{CCB}$ was identified as a promising strategy for $\mathrm{AD}$ prevention (we depicted a schematic diagram of mechanism here, see Fig. 4). One RCT found beneficial cerebrovascular effects of calcium antagonists on $\mathrm{AD}$ [35]. However, one recent MR analysis have showed that lowering SBP via AHMs is unlikely to affect the risk of developing $\mathrm{AD}$, and if specific AHM classes do reduce the risk of $\mathrm{AD}$, the mechanism may not be via SBP pathway [12]. Actually, studies have pointed out that some drugs, acting through calcium channel blocking mechanisms, have protective effects on $\mathrm{AD}$, independently of BP lowering [36]. For example, the intracellular buildup of calcium in neurons can be neurotoxic and thus CCB might result in neuroprotection [37].
Although, the underlying mechanism mediating the protective effects of AHMs on $A D$ remains unclear and warrants further research, MR analyses surely hold huge promise in the era of large-scale genetic epidemiology to identify risk or protective factors. Associations detected between AHMs (including $\mathrm{CCB}$ ) and $\mathrm{AD}$ risk undertaken by large-scale analyses should prove powerful for future studies that wish to unravel causal relationships between complex traits [29].

\section{Limitations}

The results of this study should be interpreted in conjunction with some limitations. First, we used genetic variants derived from a study with a relatively large sample size which were strongly associated with BP to avoid weak instrument problems, but our finding may still be affected by weak instrument bias [26]. Second, we were limited by the fact that MR explores the effect of lifelong exposure, whereas drugs typically have much shorter periods of exposure and BP may have age-dependent effects. The effect sizes that we have estimated will not represent the associations between critical periods of exposure and the outcome [38]. This can also be particularly problematic if the protein target of a drug is beneficial at one point during the life course and harmful at another. Thus, further work, especially RCTs, is recommended to investigate the pathways from $\mathrm{BP} /$ AHMs to AD and to explore how the effect varies with age. Third, as drug target models only focus on-target effects of the specific therapeutics, our genetic results for drug targets cannot reflect the pharmacokinetics of drug use. Thus, the associations between the drugs and the outcome cannot be fully reflected by the present analysis. Fourth, though we chose a liberal LD clumping threshold $\left(R^{2}<0.4\right)$ when selecting the variants associated with AHMs according to previously published approaches, this threshold introduced several dependent

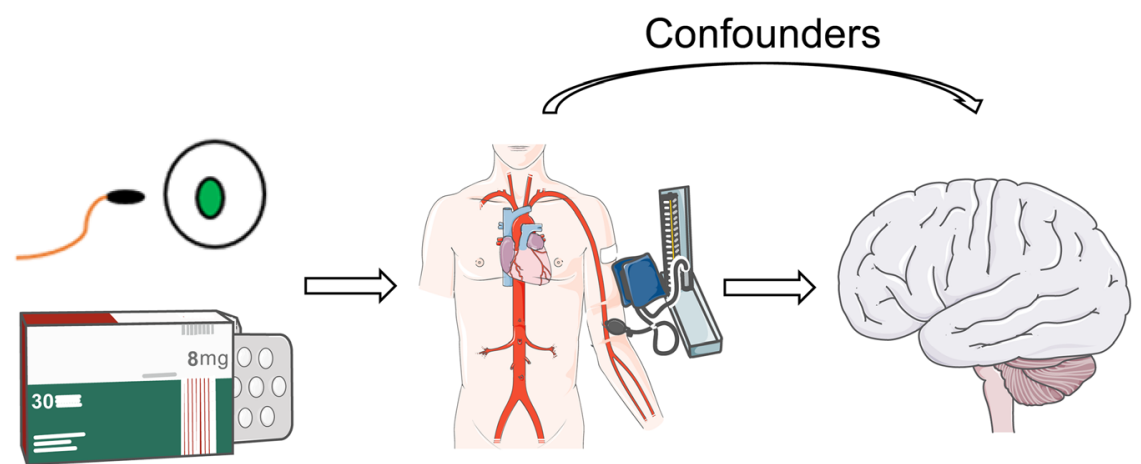

Genetic variants for AHMs $\quad$ SBP Alzheimer's disease

Fig. 4 Conceptual framework for the MR analysis of AHMs and risk of AD. Abbreviations: SBP, systolic blood pressure; AHMs, antihypertensive medications 
variants. We further employed more stringent thresholds $\left(R^{2}<0.1\right.$ and $\left.R^{2}<0.001\right)$; these results inferred the possibility that a single locus with multiple SNPs might partly drive the association. Therefore, the positive associations of AHMs, including CCB with reduced AD risk, should be interpreted with great caution. Fifth, we failed to explore the protective effects of other AHM classes, including ACEI, ARB, BB, and thiazide diuretics. These null results did not mean that there were no protective effects of these medications, given that the limited number of included SNPs failed to offer sufficient statistical power to perform meaningful analyses. Future studies encompassing larger GWAS datasets for BP might identify more variants and offer deeper insights into the effects of different classes of BP lowering agents on AD. Sixth, the estimated effect of BP level on AD risk, which is associated with a high risk of mortality, may be susceptible to survival bias. Last, since all of participants are of European ancestry, the results of this study are not necessarily valid for other ethnic groups.

\section{Conclusions}

In this two-sample MR study, we examined the effects of BP level and lowering BP via AHMs on AD using genetic variants. We provided evidence that inherited exposure to $\mathrm{BP}$ lowering through AHMs was associated with lower a risk of $\mathrm{AD}$, and $\mathrm{CCB}$ might be a promising strategy for $\mathrm{AD}$ prevention. Evidence showed that there is an effect of AHMs on $\mathrm{AD}$, which may be partly mediated by the mechanism of the BP-lowering effect. Our results complement the findings from observational studies and warrant further investigation for the development of potential AD preventive strategies targeting BP control.

\section{Supplementary Information}

The online version contains supplementary material available at https://doi. org/10.1186/s13195-021-00782-y.

Additional file 1. Demographic characteristics of included GWASes used in the present MR analysis.

Additional file 2. Drug classes, substances and targets with their DrugBank ID.

Additional file 3. Genome-wide significant and independent SNPS that were used as instruments for SBP.

Additional file 4. Genome-wide significant and independent SNPS that were used as instruments for DBP.

Additional file 5. Genome-wide significant and independent SNPS that were used as instruments for PP.

Additional file 6. SNPs that fulfilled our selection criteria to be used as proxies for the effects for AHM classes.

Additional file 7. Leave-one-out plots.

Additional file 8. MR results for the casual relationships between AHMs and $A D$ using a $L D R^{2}<0.1$.

Additional file 9. MR results for the casual relationships between AHMs and $A D$ using a LD $R^{2}<0.001$.

\section{Abbreviations}

AD: Alzheimer's disease; AHMs: Antihypertensive medications;

RCT: Randomized clinical trials; MR: Mendelian randomization; SBP: Systolic blood pressure; SNP: Single nucleotide polymorphisms; GWAS: Genome-wide association study; UKB: UK Biobank; ICBP: International Consortium of Blood Pressure; DBP: Diastolic BP; ACEl: Angiotensin converting enzyme inhibitors; ARB: Angiotensin receptor blockers; BB: $\beta$-Blockers; CCB: Calcium channel blockers; LD: Linkage disequilibrium; IGAP: International Genomics of Alzheimer's Project; ADGC: Alzheimer Disease Genetics Consortium; CHARGE: Cohorts for Heart and Aging Research in Genomic Epidemiology Consortium; EADI: The European Alzheimer's Disease Initiative; GERAD/ PERADES: Genetic and Environmental Risk in AD/Defining Genetic, Polygenic and Environmental Risk for Alzheimer's Disease Consortium; IV: Instrumental variable; IVW: Inverse variance weighted; OR: Odds ratio; Cl: Confidence interval; PRS: Polygenic risk scores; WMHV: White matter hyperintensity volume; VBI: Vascular brain injury

\section{Acknowledgements}

This work was made possible by the generous sharing of GWAS summary statistics. We thank the International Genomics of Alzheimer's Project (IGAP) for providing summary results data for these analyses. The investigators within IGAP contributed to the design and implementation of IGAP and/or provided data but did not participate in analysis or writing of this report. IGAP was made possible by the generous participation of the control subjects, the patients, and their families. Thei-Select chips were funded by the French National Foundation on Alzheimer's disease and related disorders. EADI was supported by the LABEX (laboratory of excellence program investment for the future) DISTALZ grant, Inserm, Institut Pasteur de Lille, Université de Lille 2, and the Lille University Hospital. GERAD was supported by the Medical Research Council (Grant No. 503480), Alzheimer's Research UK (Grant No. 503176), the Wellcome Trust (Grant $n^{\circ}$ 082604/2/07/Z) and German Federal Ministry of Education and Research (BMBF): Competence Network Dementia (CND) grant n 01G10102, 01Gl0711, 01GI0420. CHARGE was partly supported by the NIH/NIA grant R01 AG033193 and the NIA AG081220 and AGES contract N01-AG-12100, the NHLBI grant R01 HL105756, the Icelandic Heart Association, and the Erasmus Medical Center and Erasmus University. ADGC was supported by the NIH/NIA grants: U01 AG032984, U24 AG021886, U01 AG016976, and the Alzheimer's Association grant ADGC-10-196728.We also thank the UK Biobank (UKB) and the International Consortium of Blood Pressure (ICBP) database for providing summary results data for these analyses. The investigators within UKB/IGBP contributed to the design and implementation of UKB/IGBP and/or provided data but did not participate in analysis or writing of this report. It was made possible by the generous participation of the control subjects, the patients, and their families.

\section{Authors' contributions}

JTY conceptualized the study, interpreted the data, and revised the manuscript. YNO, YXY analyzed and interpreted the data, drafted and revised the manuscript, did the statistical analysis, and prepared all the figures. XNS, YHM, and SDC contributed to manuscript revision. QD and LT interpreted the data and revised the manuscript. All authors contributed to the writing and revisions of the paper and approved the final version.

\section{Funding}

This study was supported by grants from the National Key R\&D Program of China (2018YFC1314702), Shanghai Municipal Science and Technology Major Project (No.2018SHZDZX01) and ZHANGJIANG LAB, Tianqiao and Chrissy Chen Institute, and the State Key Laboratory of Neurobiology and Frontiers Center for Brain Science of Ministry of Education, Fudan University.

\section{Availability of data and materials}

The analyses for this study were based on publicly available summary statistics. The datasets supporting the conclusions of this article are included within the article and its additional files.

\section{Ethics approval and consent to participate}

Written informed consent was obtained from study participants or caregivers. Study protocols for all populations were approved by the appropriate Institutional review boards. 


\section{Consent for publication}

Not applicable.

\section{Competing interests}

The authors declare that they have no competing interests.

Received: 29 November 2020 Accepted: 1 February 2021

Published online: 09 February 2021

\section{References}

1. Livingston G, Sommerlad A, Orgeta V, Costafreda SG, Huntley J, Ames D, et al. Dementia prevention, intervention, and care. Lancet. 2017;390: 2673-734.

2. Qiu C, Winblad B, Fratiglioni L. The age-dependent relation of blood pressure to cognitive function and dementia. Lancet Neurol. 2005:4:487-99.

3. Xu W, Tan L, Wang HF, et al. Meta-analysis of modifiable risk factors for Alzheimer's disease. J Neurol Neurosurg Psychiatry. 2015;86:1299-306.

4. Ding J, Davis-Plourde KL, Sedaghat S, Tully PJ, Wang W, Phillips C, et al. Antihypertensive medications and risk for incident dementia and Alzheimer's disease: a meta-analysis of individual participant data from prospective cohort studies. Lancet Neurol. 2020;19:61-70.

5. Lawlor B, Segurado R, Kennelly S, Olde Rikkert MGM, Howard R, Pasquier F, et al. Nilvadipine in mild to moderate Alzheimer disease: a randomised controlled trial. Plos Med. 2018:15:e1002660.

6. Walker VM, Davey Smith G, Davies NM, Martin RM. Mendelian randomization: a novel approach for the prediction of adverse drug events and drug repurposing opportunities. Int J Epidemiol. 2017;46:2078-89.

7. Davies NM, Holmes MV, Davey SG. Reading Mendelian randomisation studies: a guide, glossary, and checklist for clinicians. BMJ. 2018;362:k601

8. Ostergaard SD, Mukherjee S, Sharp SJ, Proitsi P, Lotta LA, Day F, et al. Associations between potentially modifiable risk factors and Alzheimer disease: a Mendelian randomization study. PLoS Med. 2015;12:e1001841 discussion e41.

9. Larsson SC, Traylor M, Malik R, Dichgans M, Burgess S, Markus HS, et al. Modifiable pathways in Alzheimer's disease: Mendelian randomisation analysis. BMJ. 2017;359:j5375.

10. Andrews SJ, Fulton-Howard B, O'Reilly P, Marcora E, Goate AM. Causal associations between modifiable risk factors and the Alzheimer's phenome. Ann Neurol. 2020:89:54-65.

11. Williams DM, Finan C, Schmidt AF, Burgess S, Hingorani AD. Lipid lowering and Alzheimer disease risk: a mendelian randomization study. Ann Neurol. 2020;87:30-9.

12. Walker VM, Kehoe PG, Martin RM, Davies NM. Repurposing antihypertensive drugs for the prevention of Alzheimer's disease: a Mendelian randomization study. Int J Epidemiol. 2019;49:1132-40.

13. Evangelou E, Warren HR, Mosen-Ansorena D, Mifsud B, Pazoki R, Gao H, et al. Genetic analysis of over 1 million people identifies 535 new loci associated with blood pressure traits. Nat Genet. 2018:50:1412-25.

14. Wright JM, Musini VM, Gill R. First-line drugs for hypertension. Cochrane Database Syst Rev. 2018:4:CD001841.

15. Wishart DS, Feunang YD, Guo AC, Lo EJ, Marcu A, Grant JR, et al. DrugBank 5.0: a major update to the DrugBank database for 2018. Nucleic Acids Res. 2018:46:D1074-D82.

16. Fishilevich S, Nudel R, Rappaport N, Hadar R, Plaschkes I, Iny Stein T, et al. GeneHancer: genome-wide integration of enhancers and target genes in GeneCards. Database (Oxford). 2017;2017:bax028.

17. Nowak C, Arnlov J. A Mendelian randomization study of the effects of blood lipids on breast cancer risk. Nat Commun. 2018;9:3957.

18. Burgess S, Ference BA, Staley JR, Freitag DF, Mason AM, Nielsen SF, et al. Association of LPA variants with risk of coronary disease and the implications for lipoprotein(a)-lowering therapies: a Mendelian randomization analysis. JAMA Cardiol. 2018;3:619-27.

19. Georgakis MK, Gill D, Webb AJS, Evangelou E. Genetically determined blood pressure, antihypertensive drug classes, and risk of stroke subtypes. Neurology. 2020;95:e353-e361.

20. Kunkle BW, Grenier-Boley B, Sims R, Bis JC, Damotte V, Naj AC, et al. Genetic meta-analysis of diagnosed Alzheimer's disease identifies new risk loci and implicates $A \beta$, tau, immunity and lipid processing. Nat Genet. 2019;51:414-30.

21. Davey Smith G, Hemani G. Mendelian randomization: genetic anchors for causal inference in epidemiological studies. Hum Mol Genet. 2014;23:R89-98.
22. Rasooly D, Patel CJ. Conducting a reproducible Mendelian randomization analysis using the $\mathrm{R}$ analytic statistical environment. Curr Protoc Human Genet. 2019;101:e82.

23. Pichler I, Del Greco MF, Gogele M, Lill CM, Bertram L, Do CB, et al. Serum iron levels and the risk of Parkinson disease: a Mendelian randomization study. Plos Med. 2013;10:e1001462.

24. Bowden J, Davey Smith G, Burgess S. Mendelian randomization with invalid instruments: effect estimation and bias detection through Egger regression. Int J Epidemiol. 2015:44:512-25.

25. Burgess S, Thompson SG. Interpreting findings from Mendelian randomization using the MR-Egger method. Eur J Epidemiol. 2017;32:377-89.

26. Burgess S, Thompson SG, Collaboration CCG. Avoiding bias from weak instruments in Mendelian randomization studies. Int J Epidemiol. 2011;40: 755-64.

27. Gabin JM, Tambs K, Saltvedt I, Sund E, Holmen J. Association between blood pressure and Alzheimer disease measured up to 27 years prior to diagnosis: the HUNT Study. Alzheimers Res Ther. 2017:9:37.

28. Ou YN, Tan CC, Shen XN, Xu W, Hou XH, Dong Q, et al. Blood pressure and risks of cognitive impairment and dementia: a systematic review and metaanalysis of 209 prospective studies. Hypertension. 2020;76:217-25.

29. Richardson TG, Harrison S, Hemani G, Davey SG. An atlas of polygenic risk score associations to highlight putative causal relationships across the human phenome. eLife. 2019;8:e43657.

30. Korologou-Linden R, Howe LD, Millard LAC, Ben-Shlomo Y, Williams DM Davey Smith G, et al. The causes and consequences of Alzheimer's disease: phenome-wide evidence from Mendelian randomization. medRxiv. 2019.12. 18.19013847

31. Lane CA, Barnes J, Nicholas JM, Sudre CH, Cash DM, Parker TD, et al. Associations between blood pressure across adulthood and late-life brain structure and pathology in the neuroscience substudy of the 1946 British birth cohort (Insight 46): an epidemiological study. Lancet Neurol. 2019;18: 942-52.

32. Rajan KB, Barnes LL, Wilson RS, Weuve J, McAninch EA, Evans DA. Blood pressure and risk of incident Alzheimer's disease dementia by antihypertensive medications and APOE epsilon4 allele. Ann Neurol. 2018; 83:935-44.

33. Kleipool EEF, Trappenburg MC, Rhodius-Meester HFM, Lemstra AW, van der Flier WM, Peters MJL, et al. Orthostatic hypotension: an important risk factor for clinical progression to mild cognitive impairment or dementia. The Amsterdam Dementia Cohort. J Alzheimer's Dis. 2019;71:317-25.

34. Group SMIftSR, Williamson JD, Pajewski NM, Auchus AP, Bryan RN, Chelune $\mathrm{G}$, et al. Effect of intensive vs standard blood pressure control on probable dementia: a randomized clinical trial. JAMA. 2019;321:553-61.

35. de Jong DLK, de Heus RAA, Rijpma A, Donders R, Olde Rikkert MGM, Gunther M, et al. Effects of nilvadipine on cerebral blood flow in patients with Alzheimer disease. Hypertension. 2019;74:413-20.

36. Lebouvier T, Chen Y, Duriez P, Pasquier F, Bordet R. Antihypertensive agents in Alzheimer's disease: beyond vascular protection. Expert Rev Neurother. 2020;20:175-87.

37. JM. Fresh evidence points to an old suspect: calcium. Science. 2007;318: 384-5.

38. Holmes MV, Ala-Korpela M, Smith GD. Mendelian randomization in cardiometabolic disease: challenges in evaluating causality. Nat Rev Cardiol. 2017;14:577-90.

\section{Publisher's Note}

Springer Nature remains neutral with regard to jurisdictional claims in published maps and institutional affiliations. 